\title{
Solution a Class of Riemann-Liouville Derivative-Composition Fractional Order Optimal Control Problems
}

\author{
Moataz Abbas Holel \\ Department of Mathematics \\ University of Mustansiriyah \\ Baghdad, Iraq
}

\begin{abstract}
This paper introduces a new direction to approximately solving a class of multi between Riemann-Liouville fractional derivative of $v \in(0,1)$, and the composition of the Caputo fractional derivative of $\alpha, \beta \in(0,1)$ with the control variable $u(t)$.In this technique; we approximate FOCPs with boundary conditions. The method is based on a spectral method using Chebyshev polynomials approximation and Clenshaw and Curtis scheme for the numerical integration of non-singular functions to evaluate both the state and control variables. Illustrative examples are included to demonstrate the validity and applicability of the suggested approaches.
\end{abstract}

Keywords: Fractional calculus, Riemann-Liouville fractional derivatives, Fractional optimal control, Boundary value problems, Chebyshev polynomials, Gauss-Lobatto nodes.

\section{Introduction}

Fractional differential equations have been the focus of many studies due to their frequent appearance in various applications in fluid mechanics, biology, physics and engineering [7].Most FDEs do not have exact analytical solutions, so approximate and numerical techniques $[12,16,20]$ must be used. Several numerical methods to solve FDEs have been given. Some numerical methods for solving fractional differential equations (FDEs) were appeared in [15], [19].The general definition of an optimal control problems requires minimization of a criterion function of the states and control inputs of the system over a set of admissible control functions. The system is subject to constrained dynamics and control variables. A general formulation and a solution scheme for FOCPs were first introduced in [2] where fractional derivatives were introduced in the Riemann-Liouville sense, and FOCP formulation was expressed using the fractional variational principle and the Lagrange multiplier technique. In [1], the fractional derivatives (FDs) of the system are approximated using the Grunwald-Letnikov definition, providing a set of algebraic equations that can be solved using numerical techniques. The problem is defined in terms of the Caputo fractional derivatives in [3] and an iterative numerical scheme is introduced to solve the problem numerically. Distributed systems are considered in [4] and eigenfunction decomposition is used to solve the problem. Ozdemir et al. [18] also use eigenfunction expansion approach to formulate an FOCP of a 2dimensional distributed system. Cylindrical coordinates for the distributed system are considered in [17].

A modified Grunwald-Letnikov approach is introduced in [9] which lead to a central difference scheme. Frederico and Torres [13], [14], using similar definitions of the FOCPs, formulated a Noether-type theorem in the general context of the fractional optimal control in the sense of Caputo and studied fractional conservation laws in FOCPs. In [22], a rational approximation of the fractional derivatives operator is used to link FOCPs and the traditional integer order optimal controls (IOOCs). In [5], Akbarian and Keyanpour studied a new approach to the numerical solution of fractional order optimal control Problems. In [21], Sweilam, T.M.Al-Ajmi and R.H.W. 
Hoppe studied numerical solution of some types of fractional optimal control problems. In this article, we introduce a formulation to a special class of FOCP: the multi-Fractional we study between Riemann-Liouville fractional derivative and the composition of the Caputo fractional derivative with the control variable $u(t)$, and boundary conditions. We find approximate solution by the spectral method. The suggested method is more accurate to approximation the state and control variables. This paper is structured as follows. In Section 2, some basic definitions and properties of fractional order calculus (R-L and Caputo fractional derivatives).In Section 3, the shifted Chebyshev polynomials are introduced and numerical approximations of CFD and RLFD using Chebyshev polynomials. In Section 4, we derive the necessary optimality conditions Composition order fractional optimal control problems. In Section 5, we give numerical examples to solve Class of RiemannLiouville derivative-composition fractional order optimal control problems. The conclusions are given in Section 6.

\section{Basic definitions and properties.}

In this section, we briefly give some definitions and properties regarding fractional derivatives allowing us to formulate a general definition of an FOCP. There are different definitions of the fractional derivative operator [2]. Definition (2.1):

i. The Left Riemann-Liouville Fractional Derivative (LRLFD) of a function $f(\tau)$ is defined as

$$
{ }_{a} D_{t}^{\alpha} f(t)=\frac{1}{\Gamma(n-\alpha)} \frac{d^{n}}{d t^{n}} \int_{a}^{t}(t-\tau)^{n-\alpha-1} f(\tau) d \tau, \quad t>a
$$

where the order of the derivative $\alpha$ satisfies $n-1<\alpha<n$.

ii. The Right Riemann-Liouville Fractional Derivative (RRLFD) of a function $f(\tau)$ is defined as

$$
{ }_{t} D_{b}^{\alpha} f(t)=\frac{1}{\Gamma(n-\alpha)}(-1)^{n} \frac{d^{n}}{d t^{n}} \int_{t}^{b}(\tau-t)^{n-\alpha-1} f(\tau) d \tau, \quad t<b .
$$

\section{Definition (2.2):}

i. The Left Caputo Fractional Derivative (LCFD) of a function $f(\tau)$ is defined as

$$
{ }_{a}^{C} D_{t}^{\alpha} f(t)=\frac{1}{\Gamma(n-\alpha)} \int_{a}^{t}(t-\tau)^{n-\alpha-1} f^{(n)}(\tau) d \tau
$$

ii. The Right Caputo Fractional Derivative (RCFD) of a function $f(\tau)$ is defined as

${ }_{t}^{C} D_{b}^{\alpha} f(t)=\frac{(-1)^{n}}{\Gamma(n-\alpha)} \int_{t}^{b}(\tau-t)^{n-\alpha-1} f^{(n)}(\tau) d \tau$,

where $\in \mathrm{N}$.

\section{Some properties of the fractional calculus are presented in details which will be needed later on:}

i) The Caputo's derivatives are linear,[8]:

$$
{ }_{a}^{C} D_{t}^{\alpha}[\lambda f(t)+\mu g(t)]=\lambda{ }_{a}^{C} D_{t}^{\alpha} f(t)+\mu{ }_{a}^{C} D_{t}^{\alpha} g(t)
$$

where $\lambda, \mu$ are real numbers.

ii) Let $0<\alpha<1, n-1<\alpha+\beta<n, n-1<\beta<n, n$ is a positive integer, $f \in C^{n}[a, t]$, and if $\left.x^{(i)}(0)=0, i=0,1, \ldots \ldots n-1.\right)$

Then one has ${ }_{a}^{C} D_{t}^{\alpha}\left({ }_{a}^{C} D_{t}^{\beta} x(t)\right)={ }_{a}^{C} D_{t}^{\beta}\left({ }_{a}^{C} D_{t}^{\alpha} x(t)\right)={ }_{a}^{C} D_{t}^{\alpha+\beta} x(t)$.

iii) $\quad{ }_{t} D_{b}^{\alpha}\left({ }_{t} D_{b}^{\beta} x(t)\right)={ }_{t} D_{b}^{\alpha+\beta} x(t)-\sum_{k=1}^{m}\left[{ }_{t} D_{b}^{\beta-k} x(t)\right]_{t=b} \frac{(b-t)^{-\alpha-k}}{\Gamma(1-\alpha-k)}$,

If $\alpha>0$ and $\beta>0$ be such that $n-1<\alpha \leq n, m-1<\beta \leq m$,

when $n=m=1$, then $0<\alpha \leq 1,0<\beta \leq 1$.

${ }_{t} D_{b}^{\alpha}\left({ }_{t} D_{b}^{\beta} x(t)\right)={ }_{t} D_{b}^{\alpha+\beta} x(t)-\left[{ }_{t} D_{b}^{\beta-1} x(t)\right]_{t=b} \frac{(b-t)^{-\alpha-1}}{\Gamma(-\alpha)}$.

iv) If , $0<\alpha<1$, we obtain,[19] 


$$
\begin{aligned}
& \int_{a}^{b} g(t) \cdot{ }_{a}^{C} D_{t}^{\alpha} f(t) d t=\int_{a}^{b} f(t) \cdot{ }_{t} D_{b}^{\alpha} g(t) d t+\left[{ }_{t} I_{b}^{1-\alpha} g(t) \cdot f(t)\right]_{a}^{b} \\
& \int_{a}^{b} g(t) \cdot{ }_{t}^{C} D_{b}^{\alpha} f(t) d t=\int_{a}^{b} f(t) \cdot{ }_{a} D_{t}^{\alpha} g(t) d t+\left[\quad{ }_{a} I_{t}^{1-\alpha} g(t) \cdot f(t)\right]_{a}^{b} .
\end{aligned}
$$

Moreover,

If $f$ is a function such that $f(a)=f(b)=0$, we have simpler formulas:

$\int_{a}^{b} g(t) \cdot{ }_{a}^{C} D_{t}^{\alpha} f(t) d t=\int_{a}^{b} f(t) \cdot{ }_{t} D_{b}^{\alpha} g(t) d t$,

$\int_{a}^{b} g(t) \cdot{ }_{t}^{C} D_{b}^{\alpha} f(t) d t=\int_{a}^{b} f(t) \cdot{ }_{a} D_{t}^{\alpha} g(t) d t$.

v) The relation between the RLFD and the CFD,[6]:

For $\alpha>0$ and $n=[\alpha]+1$, the Riemann-Liouville and Caputo fractional derivatives are related by the following formulas:

$$
\begin{aligned}
& { }_{a} D_{t}^{\alpha} f(t)={ }_{a}^{C} D_{t}^{\alpha} f(t)+\sum_{k=0}^{n-1} \frac{f^{(k)}(a)}{\Gamma(k+1-\alpha)}(t-a)^{k-\alpha}, \\
& { }_{t} D_{b}^{\alpha} f(t)={ }_{t}^{C} D_{b}^{\alpha} f(t)+\sum_{k=0}^{n-1} \frac{f^{(k)}(b)}{\Gamma(k+1-\alpha)}(b-t)^{k-\alpha} .
\end{aligned}
$$

vi) The constant function and power function of the Caputo's derivative, is:

- ${ }_{a}^{C} D_{t}^{\alpha} C=0$, where $C$ is a constant,

- $\quad{ }_{a}^{C} D_{t}^{\alpha}(t-a)^{\beta}=\left\{\begin{array}{lll}0, & \text { for } \quad \beta \in N_{0} & \text { and } \beta<\lceil\alpha\rceil, \\ \frac{\Gamma(\beta+1)}{\Gamma(\beta-\alpha+1)}(t-a)^{\beta-\alpha}, & \text { for } \beta \in N_{0} & \text { and } \beta \geq\lceil\alpha\rceil,\end{array}\right.$

we use $\lceil\alpha\rceil$ to denote the smallest integer greater than or equal to $\alpha$ and $N_{0}=\{0,1,2, \ldots\}$ Recall that for $\alpha \in N,[5]$.

\section{The definition and properties of the shifted Chebyshev polynomials}

The well-known Chebyshev polynomials are defined on the interval $[-1,1]$ and can be determined by the followingrecurrence formula [11]:

$T_{n}(t)=2 t T_{n-1}(t)-T_{n-2}(t), \quad n=2,3 \ldots$.

The Chebyshev polynomials can be expanded in power series as, [5]:

$T_{n}(t)=\frac{n}{2} \sum_{m=0}^{\lfloor n / 2\rfloor}(-1)^{m} \frac{(n-m-1) !}{(m) !(n-2 m) !}(2 t)^{n-2 m}$,

where $\lfloor n / 2\rfloor$ denotes the integral part of $n / 2$, with $T_{0}(t)=1, T_{1}(t)=t$.

The Chebyshev polynomials $T_{n}(t) \quad(n=0,1,2, \ldots)$ are orthogonal under integration over $[-1,1]$ with the weighting function $w(t)=1 / \sqrt{1-t^{2}}$, with orthogonally condition:

$\int_{-1}^{1} \frac{T_{n}(t) T_{m}(t)}{\sqrt{1-t^{2}}} d t=\left\{\begin{array}{lll}0 & \text { if } & n \neq m, \\ \frac{\pi}{2} & \text { if } & n=m \in N, \\ \pi & \text { if } & n=m=0 .\end{array}\right.$

In order to use these polynomials on the interval $[0, L]$ we define the so called shiftedpseudo-spectral Chebyshev polynomials by introducing the change of variable $\quad z=\frac{2 t}{L}-1$.

The shifted Chebyshev polynomials are defined as,[6]:

$T_{n}^{p}(t)=T_{n}\left(\frac{2 t}{L}-1\right), \quad$ where $\quad T_{0}^{p}(t)=1, T_{1}^{p}(t)=\frac{2 t}{L}-1$.

The analytic form of the shifted pseudo-spectral Chebyshev polynomial $T_{n}^{p}(t)$ of degree $n$ is given by, [2]:

$T_{n}^{p}(t)=n \sum_{k=0}^{n}(-1)^{n-k} \frac{2^{2 k}(n+k-1) !}{(2 k) !(n-k) ! L^{k}} t^{k}, \quad n=1,2 \ldots$,

Where, $T_{n}^{p}(0)=(-1)^{n}$, and $T_{n}^{p}(L)=1$.

Let the shifted Chebyshev polynomials $T_{n}\left(\frac{2 t}{L}-1\right)$ be denoted by $T_{n}^{p}(t)$, satisfying the orthogonality relation: 
$\int_{0}^{L} T_{n}^{p}(t) T_{m}^{p}(t) w^{p}(t) d t=\delta m$,

with the weight function $w^{p}(t)=\frac{1}{\sqrt{L t-t^{2}}}, \delta m=\frac{b_{k}}{2} \pi, b_{0}=2, b_{k}=1$ for $k \geq 1$.

A function $x(t) \in L^{2}([0, L])$ i.e. (square integrable in $\left.[0, L]\right)$ can be expressed in terms of shifted Chebyshev polynomials as:

where the coefficients $c_{n}$ are given by

$$
x(t)=\sum_{j=0}^{\infty} c_{n} T_{n}^{p}(t)
$$

$$
c_{n}=\frac{1}{\delta n} \int_{0}^{L} x(t) T_{n}^{p}(t) w^{p}(t) d t, \quad n=0,1, \ldots
$$

\subsection{The Chebyshev-Gauss Lobatto points.}

We choose the Chebyshev-Gauss Lobatto points associated with the interval $[0, L]$,

$$
t_{r}=\frac{L}{2}-\frac{L}{2} \cos \left(\frac{\pi r}{N}\right), r=0,1, \ldots N .
$$

These grids can be written as $L<x_{N}<x_{N-1} \ldots<x_{1}<x_{0}=0$.

Clenshaw and Curtis [5], introduced an approximation of the function $x(t)$, as follows

$$
x_{N}(t)=\sum_{n=0}^{N} " a_{n} T_{n}^{p}(t), a_{n}=\frac{2}{N} \sum_{r=0}^{N} " x\left(t_{r}\right) T_{n}^{p}\left(t_{r}\right) .
$$

where the $(")$ on the summation means that the first and last terms are to be taken with a factor $(1 / 2)$.

\section{Theorem (3.1.1), [6]:}

The fractional derivative of order $\alpha$ in the Caputo sense for thefunction $x(t)$ at the point $t_{s}$ is given by

${ }_{0}^{C} D_{t}^{\alpha} x_{N}\left(t_{s}\right)=\sum_{r=0}^{N} d_{s, r}^{(\alpha)} x\left(t_{r}\right), \quad \alpha>0$,

Such that

$d_{s, r}^{(\alpha)}=\frac{4 \theta_{r}}{N} \sum_{n=\lceil\alpha\rceil}^{N} \sum_{j=0}^{N} \sum_{k=\lceil\alpha\rceil}^{n} \frac{n \theta_{n}}{b_{j}} \frac{(-1)^{n-k}(n+k-1) ! \Gamma\left(k-\alpha+\frac{1}{2}\right) T_{n}^{p}\left(t_{r}\right) T_{j}^{p}\left(t_{s}\right)}{L^{\alpha} \Gamma\left(k+\frac{1}{2}\right)(n-k) ! \Gamma(k-\alpha-j+1) \Gamma(k-\alpha+j+1)}$,

where

$s, r=0,1,2, \ldots, N$ with $\theta_{0}=\theta_{N}=\frac{1}{2}, \theta_{i}=1 \forall i=1,2, \ldots, N-1$.

Theorem (3.1.2), [6]:

Let ${ }_{0}^{C} D_{t}^{\alpha} x_{N}(t)$ be the approximation of the fractional derivative ${ }_{0}^{C} D_{t}^{\alpha}$ of the function $x(t)$ as given by (20). Then it holds

$\left\|{ }_{0}^{C} D_{t}^{\alpha} x(t)-{ }_{0}^{C} D_{t}^{\alpha} x_{N}(t)\right\|_{2} \leq \sum_{n=0}^{N \prime \prime} a_{n} \Omega_{n}\left(\frac{F\left(t^{k-\alpha} ; T_{0}^{p}, \ldots, T_{N}^{p}\right)}{F\left(T_{0}^{p}, \ldots, T_{N}^{p}\right)}\right)^{\frac{1}{2}}$,

where

$\Omega_{n}=\sum_{k=[\alpha\rceil}^{n} \frac{(-1)^{n-k} 2 n(n+k-1) ! \Gamma\left(k-\alpha+\frac{1}{2}\right)}{b_{j} L^{\alpha} \Gamma\left(k+\frac{1}{2}\right)(n-k) ! \Gamma(k-\alpha-j+1) \Gamma(k-\alpha+j+1)}$,

$F\left(x ; y_{1}, y_{2}, \ldots y_{n}\right)=\left|\begin{array}{cccc}\langle x, x\rangle & \left\langle x, y_{1}\right\rangle & \cdots & \left\langle x, y_{n}\right\rangle \\ \left\langle y_{1}, x\right\rangle & \left\langle y_{1}, y_{1}\right\rangle & \cdots & \left\langle y_{1}, y_{n}\right\rangle \\ \vdots & \vdots & \ddots & \vdots \\ \left\langle y_{n} ; x\right\rangle & \left\langle y_{n} ; y_{1}\right\rangle & \cdots & \left\langle y_{n} ; y_{n}\right\rangle\end{array}\right|$ 


\subsection{Approximation of the right Riemann-Liouville fractional derivative.}

Using right Caputo fractional derivative (RCFD) of a function $f(\tau)$ is defined in (4) when

$0<\alpha<1$ and $f(t) \in A C[a, b]$,

${ }_{s}^{c} D_{b}^{\alpha} f(s)=\frac{-1}{\Gamma(1-\alpha)} \int_{s}^{b}(t-s)^{-\alpha} f^{\prime}(t) d t$,

and from (10), when $0<\alpha<1$, (that is $n=1$ ) we have

$$
{ }_{s} D_{b}^{\alpha} f(s)=\frac{f(b)}{\Gamma(1-\alpha)}(b-s)^{-\alpha}+{ }_{s}^{c} D_{b}^{\alpha} f(s),
$$

Use (25) in (26) to obtain:

$$
{ }_{s} D_{b}^{\alpha} f(s)=\frac{f(b)}{\Gamma(1-\alpha)}(b-s)^{-\alpha}+\frac{1}{\Gamma(1-\alpha)} \int_{s}^{b}(t-s)^{-\alpha} f^{\prime}(t) d t
$$

Let $f$ be a sufficiently smooth function in $[0, b]$ and let $J(s ; f)$ be defined as follows

$J(s ; f)=\int_{s}^{b}(t-s)^{-\alpha} f^{\prime}(t) d t, 0<s<b$.

Substitution (28) in (27) we deduce

$$
{ }_{s} D_{b}^{\alpha} f(s)=\frac{f(b)}{\Gamma(1-\alpha)}(b-s)^{-\alpha}+\frac{J(s ; f)}{\Gamma(1-\alpha)} .
$$

Now, we approximate $f(t), 0 \leq t \leq b$, by a sum of shifted Chebyshev polynomials

$T_{k}\left(\frac{2 t}{b}-1\right)$ according to

$f(t) \approx p_{N}(t)=\sum_{k=0}^{N}{ }^{\prime \prime} a_{k} T_{k}\left(\frac{2 t}{b}-1\right), a_{k}=\frac{2}{N} \sum_{j=0}^{N "} f\left(t_{j}\right) T_{k}\left(\frac{2 t_{j}}{b}-1\right)$,

where

$t_{j}=\frac{b}{2}-\frac{b}{2} \cos \left(\frac{\pi j}{N}\right), j=0, \ldots \ldots, N$, and obtain

$J(s ; f) \approx J\left(s ; p_{N}\right)=\int_{s}^{b} p_{N}{ }^{\prime}(t)(t-s)^{-\alpha} d t$.

\section{Lemma (3.2.1), [6]:}

Let $p_{N}$ be the polynomial of degree $N$ as given by (30), Then there exists a polynomial $F_{N-1}$ of degree $N-1$ such that

$\int_{S}^{x}\left[p_{N}{ }^{\prime}(t)-p_{N}{ }^{\prime}(s)\right](t-s)^{-\alpha} d t=\left[F_{N-1}(x)-F_{N-1}(s)\right](x-s)^{1-\alpha}$.

\section{Proof:}

Let $p_{N}{ }^{\prime}(t)-p_{N}{ }^{\prime}(s)$ be expanded in a Taylor series at $t=s$

$p_{N}{ }^{\prime}(t)-p_{N}{ }^{\prime}(s)=\sum_{k=1}^{N-1} A_{k}(s)(t-s)^{k}$.

Then,

$$
\begin{gathered}
\int_{S}^{x}\left[p_{N}{ }^{\prime}(t)-p_{N}{ }^{\prime}(s)\right](t-s)^{-\alpha} d t=\sum_{k=1}^{N-1} A_{k}(s) \int_{s}^{x}(t-s)^{k-\alpha} d t \\
=\left[(t-s)^{1-\alpha} \sum_{k=1}^{N-1} \frac{A_{k}(s)(t-s)^{k}}{k-\alpha+1}\right]_{S}^{x} .
\end{gathered}
$$

The assertion follows, if we choose $F_{N-1}(x)=\sum_{k=0}^{N-1} \frac{A_{k}(s)(x-s)^{k}}{k-\alpha+1}$, with an arbitrary constant $A_{0}(s)$.

In view of (32) we have

$J\left(s ; p_{N}\right)=\int_{s}^{b} p_{N}{ }^{\prime}(t)(t-s)^{-\alpha} d t=\left[\frac{p_{N}{ }^{\prime}(s)}{1-\alpha}+\left[F_{N-1}(b)-F_{N-1}(s)\right]\right](b-s)^{1-\alpha}$

Moreover, ${ }_{s} D_{b}^{\alpha} f(s)$ can be approximated by means of

$$
{ }_{s} D_{b}^{\alpha} f(s) \approx \frac{f(b)}{\Gamma(1-\alpha)}(b-s)^{-\alpha}+\frac{J\left(s ; p_{N}\right)}{\Gamma(1-\alpha)} .
$$

We express $F_{N-1}(t)$ in (33) by a sum of Chebyshev polynomials and provide the recurrence relation satisfied by the Chebyshev coefficients. Differentiating both sided of (32) with respect to $x$ yields 
when

$$
\begin{gathered}
{\left[p_{N}{ }^{\prime}(x)-p_{N}{ }^{\prime}(s)\right](x-s)^{-\alpha}=} \\
F_{N-1}{ }^{\prime}(x)(x-s)^{1-\alpha}+\left[F_{N-1}(x)-F_{N-1}(s)\right](1-\alpha)(x-s)^{-\alpha},
\end{gathered}
$$

$p_{N}^{\prime}(x)-p_{N}^{\prime}(s)=F_{N-1}{ }^{\prime}(x)(x-s)+\left[F_{N-1}(x)-F_{N-1}(s)\right](1-\alpha)$.

To evaluate $F_{N-1}(s)$ in (33), we expand $F_{N-1}{ }^{\prime}(x)$ in terms of the shifted chebyshev polynomials

$F_{N-1}^{\prime}(x)=\sum_{k=0}^{N-2}{ }^{\prime} b_{k} T_{k}\left(\frac{2 x}{b}-1\right), 0 \leq x \leq b$,

where the ( $\left.{ }^{\prime}\right)$ on the summation means that the first term is to be taken with a factor $(1 / 2)$.

$F_{N-1}(x)-F_{N-1}(s)=\frac{b}{4} \sum_{k=1}^{N-1} \frac{b_{k-1}-b_{k+1}}{k}\left(T_{k}\left(\frac{2 x}{b}-1\right)-T_{k}\left(\frac{2 s}{b}-1\right)\right)$,

where $b_{N-1}=b_{N}=0$. On the other hand, we have

$$
(x-s) F_{N-1}^{\prime}(x)=\frac{b}{2} F_{N-1}{ }^{\prime}(x)\left[\left(\frac{2 x}{b}-1\right)-\left(\frac{2 s}{b}-1\right)\right] .
$$

By using the relation $T_{k+1}(v)+T_{k-1}(v)=2 v T_{k}(v)$ and from (36), it follows that

$(x-s) F_{N-1}{ }^{\prime}(x)=\frac{b}{4} \sum_{k=1}^{N-1}{ }^{\prime}\left(b_{k+1}-2\left(\frac{2 s}{b}-1\right) b_{k}+b_{k-1}\right) T_{k}\left(\frac{2 x}{b}-1\right)$,

Such that $b_{-1}=b_{1}$

Let $p_{N}{ }^{\prime}(x)=\sum_{k=0}^{N-1} \quad c_{k} T_{k}\left(\frac{2 x}{b}-1\right)$.

Inserting $F_{N-1}(x)-F_{N-1}(s)$ and $(x-s) F_{N-1}{ }^{\prime}(x)$ as given (37) and (38) into (35) and taking (39) into account, we get

$\left(1-\frac{1-\alpha}{k}\right) b_{k+1}-2\left(\frac{2 s}{b}-1\right) b_{k}+\left(1+\frac{1-\alpha}{k}\right) b_{k-1}=\frac{4}{b} c_{k}, \quad 1 \leq k$

The Chebyshev coefficients $c_{k}$ of $p_{N}{ }^{\prime}(x)$ as given by (39) can be evaluated by integrating and comparing it with equation (30):

$c_{k-1}=c_{k+1}+\frac{4 k}{b} a_{k}, k=N, N-1, \ldots, 1$,

with starting values $c_{N}=c_{N+1}=0$, where $a_{k}$ are the Chebyshev coefficients of $p_{N}(x)$.

\section{The necessary optimality conditions of Riemann-Liouville Derivative-Composition Fractional Order Optimal Control Problems:}

$$
M_{a} D_{t}^{v} x(t)+N_{a}^{C} D_{t}^{\alpha}\left({ }_{a}^{c} D_{t}^{\beta} x(t)\right)=f(t, x(t), u(t))
$$

In this type, we introduce the system of the type multi of the Riemann-Liouville fractional derivative of $v \in(0,1)$, and the composition of the Caputo fractional derivative of $\alpha, \beta \in(0,1)$ with the control variable $u(t)$. The necessary optimality conditions of this type introduce as follows:

Let $f_{0}, f:\left[a, \infty\left[\times \mathbb{R}^{2} \rightarrow \mathbb{R}\right.\right.$ be two differentiable functions, and let $v, \alpha$ and $\beta$ be real numbers and $v, \alpha$ and $\beta \in$ $(0,1)$. We consider a general multi-order Fractional Optimal Control Problem:

$$
\operatorname{minimize} \mathrm{J}(x, u, T)=\int_{a}^{T} f_{0}(t, x(t), u(t)) d t,
$$

subject to the RLD-composition fractional dynamical system

$$
M_{a} D_{t}^{v} x(t)+N_{a}^{C} D_{t}^{\alpha}\left({ }_{a}^{C} D_{t}^{\beta} x(t)\right)=f(t, x(t), u(t)),
$$

and the boundary conditions

$x(a)=x_{a}, \quad x(T)=x_{T}, \quad x(a)=x_{a}$ and,$x(T)$ is not specified 
where $M, N \neq 0, T, x_{a}$ and $x_{T}$ are fixed real numbers.

\section{Theorem (4.1):}

If $(x, u, T)$ is a minimizer of (42), (43) and (44), then there exists a function $\lambda(t)$ for which $(x, u, T)$ satisfies the RLD-composition fractional optimality conditions:

i) the Hamiltonian system defined as

$$
\begin{aligned}
M_{t}^{C} D_{b}^{v} \lambda(t)+N{ }_{t} D_{b}^{\alpha}\left({ }_{t} D_{b}^{\beta} \lambda(t)\right) & =\frac{\partial H}{\partial x}(t, x(t), u(t), \lambda(t)) \\
M_{a} D_{t}^{v} x(t)+N_{a}^{C} D_{t}^{\alpha}\left({ }_{a}^{C} D_{t}^{\beta} x(t)\right) & =\frac{\partial H}{\partial \lambda}(t, x(t), u(t), \lambda(t))
\end{aligned}
$$

the stationary condition

ii) $\quad \frac{\partial H}{\partial u}(t, x(t), u(t), \lambda(t))=0$

\section{Proof:}

$$
\text { for all } t \in[a, T] \text {; }
$$

We consider the following multi-composite fractional optimal control problem

$$
\begin{gathered}
\operatorname{minimize} \mathrm{J}(x, u, T)=\int_{a}^{T} f_{0}(t, x(t), u(t)) d t, \\
M_{a}^{C} D_{t}^{\alpha}(t)+N_{a}^{C} D_{t}^{\beta}\left({ }_{a}^{C} D_{t}^{\mu} x(t)\right)=f(t, x(t), u(t)),
\end{gathered}
$$

Thus, $\quad J^{*}(x, u, T, \lambda)=\int_{a}^{T}\left\{\left(f_{0}(t, x(t), u(t))+\lambda(t) \cdot\left[f(t, x(t), u(t))-M_{a} D_{t}^{v} x(t)+N_{a}^{C} D_{t}^{\alpha}\left({ }_{a}^{C} D_{t}^{\beta} x(t)\right)\right]\right\} d t\right.$.

Suppose that,

$F=f_{0}(t, x(t), u(t))+\lambda(t) f(t, x(t), u(t))-M \lambda(t){ }_{a} D_{t}^{v} x(t)-N \lambda(t){ }_{a}^{C} D_{t}^{\alpha}\left({ }_{a}^{C} D_{t}^{\beta} x(t)\right)$.

Evaluating the Euler equation for $x(t)$,

$\frac{\partial F}{\partial x(t)}-\frac{d}{d t}\left(\frac{\partial F}{\partial x^{\cdot}(t)}\right)=0$,

From property (7), we get

$F=f_{0}(t, x(t), u(t))+\lambda(t) f(t, x(t), u(t))-M x(t) \cdot{ }_{t}^{C} D_{b}^{v} \lambda(t)-N x(t) \cdot{ }_{t} D_{b}^{\alpha}\left({ }_{t} D_{b}^{\beta} \lambda(t)\right)$.

Then from (49) which gives

$\frac{\partial f_{0}}{\partial x(t)}+\lambda(t) \frac{\partial f}{\partial x(t)}-M{ }_{t}^{C} D_{b}^{v} \lambda(t)-N{ }_{t} D_{b}^{\alpha}\left({ }_{t} D_{b}^{\beta} \lambda(t)\right)=0$,

From (50), we get $\frac{d}{d t}\left(\frac{\partial F}{\partial x^{\cdot}(t)}\right)=0$,

$M_{t}^{C} D_{b}^{v} \lambda(t)+N{ }_{t} D_{b}^{\alpha}\left({ }_{t} D_{b}^{\beta} \lambda(t)\right)=\frac{\partial f_{0}}{\partial x(t)}+\lambda(t) \frac{\partial f}{\partial x(t)}$.

Now, evaluating Euler's equation for $\lambda(t)$; that is,

$\frac{\partial F}{\partial \lambda(t)}-\frac{d}{d t}\left(\frac{\partial F}{\partial \lambda^{\cdot}(t)}\right)=0$

Then according to (53) for (48) which gives:

$M_{a} D_{t}^{v} x(t)+N_{a}^{C} D_{t}^{\alpha}\left({ }_{a}^{C} D_{t}^{\beta} x(t)\right)=\frac{\partial f_{0}}{\partial \lambda(t)}+\lambda(t) \frac{\partial f}{\partial \lambda(t)} .0$,

Finally, the Euler's equation for $u(t)$; that is,

$\frac{\partial F}{\partial u(t)}-\frac{d}{d t}\left(\frac{\partial F}{\partial u \cdot(t)}\right)=0$

From (48) it is clear that $\frac{d}{d t}\left(\frac{\partial F}{\partial u^{\cdot}(t)}\right)=0$, then we get 
$\frac{\partial F}{\partial u(t)}=\frac{\partial f_{0}}{\partial u(t)}+\lambda(t) \frac{\partial f}{\partial u(t)}=0$

From (52), (54) and (56) we have that,

$$
\begin{aligned}
M_{t}{ }_{t} D_{b}^{v} \lambda(t)+N{ }_{t} D_{b}^{\alpha}\left({ }_{t} D_{b}^{\beta} \lambda(t)\right) & =\frac{\partial H}{\partial x}(t, x(t), u(t), \lambda(t)) \\
M_{a} D_{t}^{v} x(t)+N_{a}{ }_{a} D_{t}^{\alpha}\left({ }_{a} D_{t}^{\beta} x(t)\right) & =\frac{\partial H}{\partial \lambda}(t, x(t), u(t), \lambda(t))
\end{aligned}
$$

$0=\frac{\partial H}{\partial u}(t, x(t), u(t), \lambda(t))$,for all $t \in[a, T]$.

\section{Illustrative Example:}

In this section, we developed the algorithm for solution the Riemann-Liouville derivative-composition fractional order optimal control problems.

Consider the following linear-quadratic of RLD fractional optimal control problem:

$\min \mathrm{J}(x, u)=J(x, u)=\int_{0}^{1} u^{2}(t) d t$,

subject to the fractional dynamic control system

$$
{ }_{0} D_{t}^{v} x(t)+{ }_{0}^{C} D_{t}^{\alpha}\left({ }_{0}^{C} D_{t}^{\beta} x(t)\right)=u(t)+\frac{6 t^{\alpha+\beta-v+1}}{\Gamma(\alpha+\beta-v+2)}-\frac{6 t^{\alpha+\beta-v}}{\Gamma(\alpha+\beta-v+1)}+\frac{\Gamma(\beta+1) t^{\beta-v}}{\Gamma(\beta-v+1)}+\frac{t^{-v}}{\Gamma(1-v)} .
$$

and the boundary conditions

$x(0)=1, x(1)=0, \quad x(0)=1$ and,$x(1)$ is not specified

The exact solution for $v=\alpha=\beta=1$ is given by:

$$
(\bar{x}(t), \bar{u}(t))=\left(\frac{6 t^{\alpha+\beta+1}}{\Gamma(\alpha+\beta+2)}-\frac{6 t^{\alpha+\beta}}{\Gamma(\alpha+\beta+1)}+t^{\beta}+1,6(t-1)\right)
$$

Now, we develop algorithm for the solution (57), (58) and (59). It is based on the necessary optimality conditions of RLD composition-order fractional optimal control from Theorem (4.1) as the following steps:

Step 1: Compute the Hamiltonian function

$H(t, x, u, \lambda)=u^{2}(t)+\lambda(t)\left(u(t)+\frac{6 t^{\alpha+\beta-v+1}}{\Gamma(\alpha+\beta-v+2)}-\frac{6 t^{\alpha+\beta-v}}{\Gamma(\alpha+\beta-v+1)}+\frac{\Gamma(\beta+1) t^{\beta-v}}{\Gamma(\beta-v+1)}+\frac{t^{-v}}{\Gamma(1-v)}\right)$.

Step 2: Derive the necessary optimality conditions of RLD composition-order fractional optimal control from Theorem (4.1):

Suppose that $M=N=1$, of the constraint (58), then

$$
\begin{aligned}
& { }_{0} D_{t}^{v} x(t)+{ }_{0}^{C} D_{t}^{\alpha}\left({ }_{0}^{C} D_{t}^{\beta} x(t)\right)=u(t)+\frac{6 t^{\alpha+\beta-v+1}}{\Gamma(\alpha+\beta-v+2)}-\frac{6 t^{\alpha+\beta-v}}{\Gamma(\alpha+\beta-v+1)}+\frac{\Gamma(\beta+1) t^{\beta-v}}{\Gamma(\beta-v+1)}+\frac{t^{-v}}{\Gamma(1-v)}, \\
& { }_{t}^{C} D_{1}^{v} \lambda(t)+{ }_{t} D_{1}^{\alpha}\left({ }_{t} D_{1}^{\beta} \lambda(t)\right)=0, \\
& u(t)=-\frac{1}{2} \lambda(t) .
\end{aligned}
$$

Step 3: Substitution control variable $u(t)$ from (64) in equation (62) to obtain:

$$
\begin{aligned}
{ }_{0} D_{t}^{v} x(t)+{ }_{0}^{C} D_{t}^{\alpha} & \left({ }_{0}^{C} D_{t}^{\beta} x(t)\right) \\
& =-\frac{1}{2} \lambda(t)+\frac{6 t^{\alpha+\beta-v+1}}{\Gamma(\alpha+\beta-v+2)}-\frac{6 t^{\alpha+\beta-v}}{\Gamma(\alpha+\beta-v+1)}+\frac{\Gamma(\beta+1) t^{\beta-v}}{\Gamma(\beta-v+1)}+\frac{t^{-v}}{\Gamma(1-v)},
\end{aligned}
$$

Using property (5) and (6) for equations (63) and (65), we get the coupled system:

$$
\left\{\begin{array}{l}
{ }_{t}^{C} D_{1}^{v} \lambda(t)+{ }_{t} D_{1}^{\alpha+\beta} \lambda(t)-\left[{ }_{t} D_{1}^{\beta-1} \lambda(t)\right]_{t=1} \frac{(1-t)^{-\alpha-1}}{\Gamma(-\alpha)}=0 \\
{ }_{0} D_{t}^{v} x(t)+{ }_{0}^{C} D_{t}^{\alpha+\beta} x(t)=-\frac{1}{2} \lambda(t)+\frac{6 t^{\alpha+\beta-v+1}}{\Gamma(\alpha+\beta-v+2)}-\frac{6 t^{\alpha+\beta-v}}{\Gamma(\alpha+\beta-v+1)}+\frac{\Gamma(\beta+1) t^{\beta-v}}{\Gamma(\beta-v+1)}+\frac{t^{-v}}{\Gamma(1-v)} .
\end{array}\right.
$$


Step 4: Using the Chebyshev expansion method, get an approximate solution of $\lambda(t)$ from (20) and $x(t)$ from (34):

$$
\sum_{r=0}^{N} d_{s, r}^{(v)} \lambda\left(t_{r}\right)+\frac{\lambda(1)}{\Gamma(1-(\alpha+\beta))}\left(1-t_{s}\right)^{-(\alpha+\beta)}+\frac{J\left(t_{s} ; p_{N}\right)}{\Gamma(1-(\alpha+\beta))}-\left[{ }_{t} D_{1}^{\beta-1} \lambda\left(t_{s}\right)\right]_{t=1} \frac{\left(1-t_{s}\right)^{-\alpha-1}}{\Gamma(-\alpha)}=0,
$$

$\frac{x(1)}{\Gamma(1-v)}\left(1-t_{s}\right)^{-(v)}+\frac{J\left(t_{s} ; p_{N}\right)}{\Gamma(1-v)}+\sum_{r=0}^{N} d_{s, r}^{(\alpha+\beta)} x\left(t_{r}\right)=-\frac{1}{2} \lambda\left(t_{s}\right)+\frac{6 t_{s}{ }^{\alpha+\beta-v+1}}{\Gamma(\alpha+\beta-v+2)}-\frac{6 t_{s}{ }^{\alpha+\beta-v}}{\Gamma(\alpha+\beta-v+1)}+\frac{\Gamma(\beta+1) t_{s}{ }^{\beta-v}}{\Gamma(\beta-v+1)}+$

$\frac{t_{s}{ }^{-v}}{\Gamma(1-v)}$.

where $d_{s, r}^{(v)}$ and $d_{s, r}^{(\alpha+\beta)}$ is defined in (21) and evaluating the results at the shifted Gauss-Lobatto nodes $t_{s}$, from (21), $s=1,2, \ldots, N-1$.

Step 5: Evaluating the results at the shifted Gauss-Lobatto nodes $t_{s}$,from (18), $s=1,2, \ldots, N-1$,and $\alpha, \beta$ and $\mu \in(0,1)$.

Let $N=2, v=0.12, \alpha=0.4, \quad \beta=0.3$.

$s=1,2, \ldots, N-1$, and since $N=2$, then $s=1 \cdot t_{1}=\frac{1}{2}-\frac{1}{2} \cos \left(\frac{\pi}{2}\right)=0.5$

From the system (68), we have

$$
\begin{array}{r}
\frac{x(1)}{\Gamma(1-0.12)}(0.5)^{-(0.12)}+\frac{J\left(t_{1} ; p_{N}\right)}{\Gamma(1-0.12)}+d_{1,0}^{(0.7)} x\left(t_{0}\right)+d_{1,1}^{(0.7)} x\left(t_{1}\right)+d_{1,2}^{(0.7)} x\left(t_{2}\right) \\
=\frac{6(0.5)^{1.5800}}{\Gamma(2.5800)}-\frac{6(0.5)^{0.5800}}{\Gamma(1.5800)}+\frac{\Gamma(1.3)(0.5)^{0.1800}}{\Gamma(1.1800)}+\frac{(0.5)^{-0.12}}{\Gamma(0.8800)},
\end{array}
$$

$(1.0013) x(1)+(0.9214) J\left(t_{1} ; p_{N}\right)+d_{1,0}^{(0.7)} x\left(t_{0}\right)+d_{1,1}^{(0.7)} x\left(t_{1}\right)+d_{1,2}^{(0.7)} x\left(t_{2}\right)=-1.0189$.

\begin{tabular}{|c|c|c|c|c|}
\hline$n$ & $j$ & $\boldsymbol{k}$ & $d_{1,0}^{(0.7)}$ & $d_{1,1}^{(0.7)}$ \\
\hline 1 & $\mathbf{0}$ & 1 & -0.8154 & $\mathbf{0}$ \\
\hline 1 & 1 & 1 & $\mathbf{0}$ & $\mathbf{0}$ \\
\hline 1 & 2 & 1 & -0.0572 & $\mathbf{0}$ \\
\hline 2 & $\mathbf{0}$ & 1 & -0.8154 & 0.8154 \\
\hline 2 & 1 & 1 & $\mathbf{0}$ & $\mathbf{0}$ \\
\hline 2 & 2 & 1 & -0.0572 & 0.0572 \\
\hline 2 & 0 & 2 & 0.7720 & -0.7720 \\
\hline 2 & 1 & 2 & $\mathbf{0}$ & $\mathbf{0}$ \\
\hline 2 & 2 & 2 & -0.0396 & 0.0396 \\
\hline \multicolumn{3}{|c|}{ sum } & -1.0128 & 0.1402 \\
\hline \multicolumn{3}{|c|}{$d_{s r}^{(\alpha)}=4 \theta_{r} / N . s u m$} & -1.0128 & 0.0701 \\
\hline
\end{tabular}

Use (21) to evaluating the results of $d_{1,0}^{(0.7)}, d_{1,1}^{(0.7)}$, and $d_{1,2}^{(0.7)}$, which is shown in table (1) as follows:

Table (1): Shows results of Caputo fractional derivative when $\alpha=0.4, \beta=0.3, \alpha+\beta=0.7$.

when $\quad r=0 \rightarrow \frac{4 \theta_{0}}{N}=\frac{4(0.5)}{2}=1$ and $\quad r=1 \rightarrow \frac{4 \theta_{1}}{N}=\frac{4(1)}{2}=2$

Since, $x \cdot(1)$ is not specified, then we have the transversality condition

$\lambda\left(t_{2}\right)=0$, We conclude from this $J\left(t_{1} ; p_{N}\right)=0$, and use the boundary conditions (59) in (69) to get $\left(t_{1}\right)$ :

$$
\begin{gathered}
(1.0013)(0)+(0.9214)(0)+(-1.0128)(1)+(0.0701) x\left(t_{1}\right)+d_{1,2}^{(0.7)}(0)=-1.0119 . \\
\rightarrow x\left(t_{1}\right)=0.0128
\end{gathered}
$$


Step 6.Computeapproximation solution of $x(t)$ at $N=2$ by (19), and $u(t)$ from (58).

$x_{2}(t)=\frac{1}{2} a_{0} T_{0}^{p}(t)+a_{1} T_{1}^{p}(t)+\frac{1}{2} a_{2} T_{2}^{p}(t)$

To find $a_{0}, a_{1}$ and $a_{2}$

$$
\begin{array}{cc}
\text { If } n=0 \rightarrow a_{0}=\sum_{r=0}^{2} " x\left(t_{r}\right) T_{0}^{p}\left(t_{r}\right), & \text { i.e. } T_{0}^{p}(t)=1 . \\
=0.5128 . & \text { i.e. } T_{1}^{p}(t)=\frac{2 t}{b}-1 . \\
\text { If } n=1 \rightarrow a_{1}=\sum_{r=0}^{2} " x\left(t_{r}\right) T_{1}^{p}\left(t_{r}\right), & \\
=-0.5 & \text { i.e. } T_{2}^{p}(t) \text { from (13). } \\
\text { If } n=2 \rightarrow a_{2}=\sum_{r=0}^{2 \prime \prime} x\left(t_{r}\right) T_{2}^{p}\left(t_{r}\right), & \\
=0.4872 . &
\end{array}
$$

\begin{tabular}{|c|c|c|c|c|}
\hline $\mathbf{t}$ & $\begin{array}{c}\alpha=0.4 \\
\beta=0.3 \\
\nu=0.12\end{array}$ & $\begin{array}{l}\alpha=1 \\
\beta=1 \\
v=1\end{array}$ & $\begin{array}{c}\alpha=0.4 \\
\beta=0.3 \\
v=0.12\end{array}$ & $\begin{array}{l}\alpha=1 \\
\beta=1 \\
v=1\end{array}$ \\
\hline & $x(t)$ & $\bar{x}(t)$ & $u(t)$ & $\bar{u}(t)$ \\
\hline $\mathbf{0}$ & 1 & 1 & $\mathbf{0}$ & -6 \\
\hline 0.1 & 1.0724 & 1.0710 & -3.8413 & -5.4000 \\
\hline 0.2 & 1.0488 & 1.0880 & -2.6746 & -4.8000 \\
\hline 0.3 & 1.0290 & 1.0570 & -2.5056 & -4.2000 \\
\hline 0.4 & 0.1323 & 0.9840 & -1.3411 & -3.6000 \\
\hline 0.5 & 0.5128 & 0.8750 & -1.1798 & -3 \\
\hline 0.6 & 0.6770 & 0.7360 & -1.0199 & -2.4000 \\
\hline 0.7 & 0.3109 & 0.5730 & -0.1404 & -1.8000 \\
\hline 0.8 & 0.3111 & 0.3920 & -0.3025 & -1.2000 \\
\hline 0.9 & 0.1075 & 0.1990 & -0.4679 & -0.6000 \\
\hline 1 & 0.6374 & $\mathbf{0}$ & $\mathbf{0}$ & $\mathbf{0}$ \\
\hline
\end{tabular}

$x_{2}(t)=1.9488 t^{2}-2.9488 t+1$.

To compute the approximation solution of the control $u(t)$, we get

$u(t)={ }_{0} D_{t}^{v} x(t)+{ }_{0}^{C} D_{t}^{\alpha}\left({ }_{0}^{C} D_{t}^{\beta} x(t)\right)-\frac{6 t^{\alpha+\beta-v+1}}{\Gamma(\alpha+\beta-v+2)}+\frac{6 t^{\alpha+\beta-v}}{\Gamma(\alpha+\beta-v+1)}-\frac{\Gamma(\beta+1) t^{\beta-v}}{\Gamma(\beta-v+1)}-\frac{t^{-v}}{\Gamma(1-v)}$.

After we derive a fractional derivation of the power function of Caputo's derivative and R-L derivative and substitute (71) in (72), we have:

$$
\begin{aligned}
u(t)=2.1707 t^{1.8800}-3.0875 t^{0.8800}+3.3407 t^{1.3}-3.2857 t^{0.3}-4.2600 t^{1.5800}+6.7308 t^{0.5800} & \\
- & 0.9716 t^{0.1800}
\end{aligned}
$$

Table (2): Shows numerical results of the exact and approximate state $x(t)$ and control $u(t)$ for $N=2$. 


\section{Figure 1:}
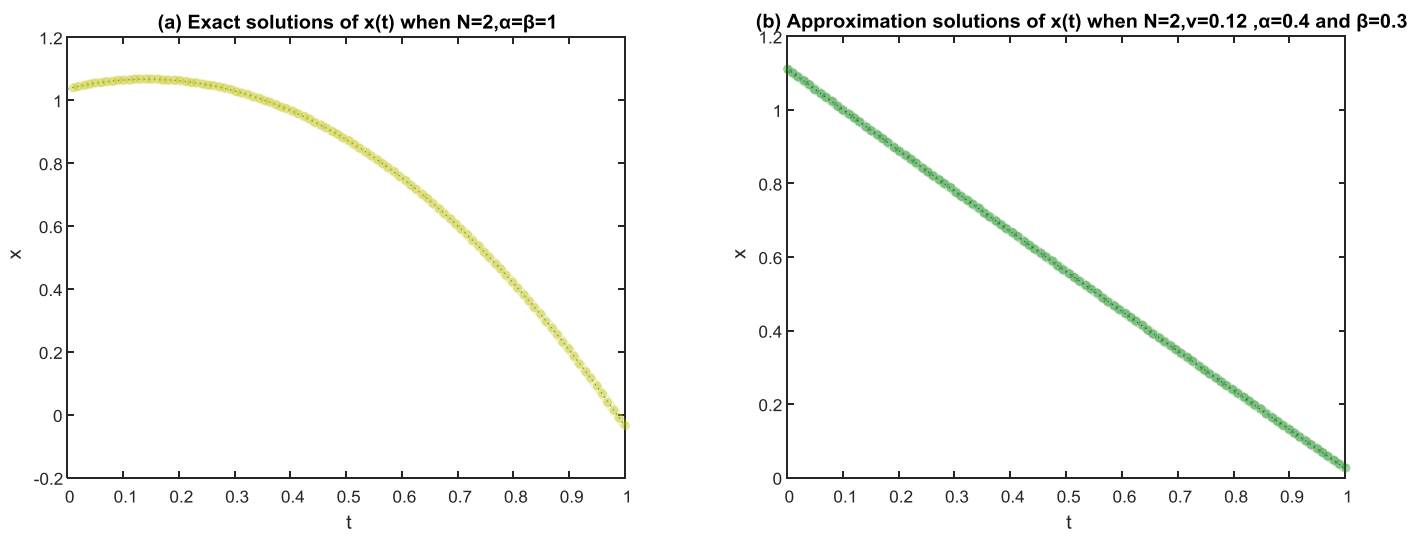

(a) Exact solution of $x(t)$ for $(N=2)$ and $v=\alpha=\beta=1$.

(b) Approximate solutions of $x(t)$ for $(N=2)$ and $v=0.12, \alpha=0.4$ and $\beta=0.3$

\section{Figure 2:}
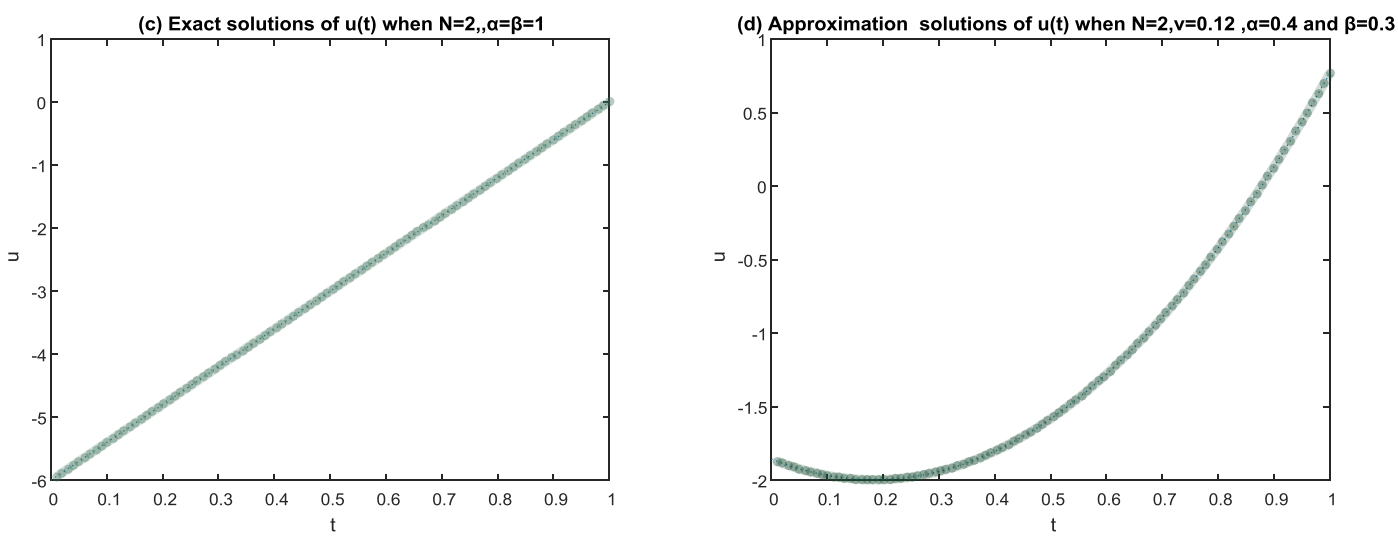

(c) Exact solution of $u(t)$ for $(N=2)$ and $\alpha=\beta=1$.

(d) Approximate solutions of $u(t)$ for $(N=2)$ and $v=0.12, \alpha=0.4$ and $\beta=0.3$.

\section{Conclusions}

In this paper, we introduced an accurate numerical scheme for solving a class of RLD composition-order fractional optimal control problems, when $\alpha=0.4, \beta=0.3$ and $v=0.12$ at $(N=2)$.In this case, the solution is approximated by Chebyshev series.

Numerical results for illustrative example show that the algorithm converge from the exact solution when $\alpha=\beta=v=1$, and we note that the convergent to the exact solution is dependent on increasing of the fractional order of derivative.

\section{References}

[1] Agrawal, Om P., and Dumitru Baleanu. " A Hamiltonian formulation and a direct numerical scheme for fractional optimal control problems." Journal of Vibration and Control13.9-10 (2007): 1269-1281.

[2] Agrawal, Om Prakash. "A general formulation and solution scheme for fractional optimal control problems." Nonlinear Dynamics 38.1-4 (2004): 323-337.

[3] Agrawal, Om P. "A quadratic numerical scheme for fractional optimal control problems." Journal of dynamic systems, measurement, and control 130.1 (2008): 011010.

[4] Agrawal, Om P. "Fractional optimal control of a distributed system using eigenfunctions." Journal of Computational and Nonlinear Dynamics 3.2 (2008): 021204. 
[5] Akbarian, T., and M. Keyanpour. " A new approach to the numerical solution of fractional order optimal control problems." Applications and Applied Mathematics 8.2 (2013): 523-534.

[6] Al-Mdallal, Qasem M., Muhammed I. Syam, and M. N. Anwar. " A collocation-shooting method for solving fractional boundary value problems." Communications in Nonlinear Science and Numerical Simulation 15.12 (2010): 3814-3822.

[7] Bagley.R. L. and TorvikP. J., " On the appearance of the fractional derivative in the behavior of real materials," J. Appl. Mech. 51 (1984), 294-298.

[8] Baleanu.D., Machado.J. A. T. andLuo, A. C. (Eds.). " Fractional dynamics and control." Springer Science \& Business Media, 2011.

[9] Baleanu.D., DefterliO.., and Agrawal.O. P. "A central difference numerical scheme for fractional optimal control problems," Journal of Vibration and Control, vol. 15, no. 4, pp.583-597, 2009.

[10] Bell, William Wallace. " Special functions for scientists and engineers." Courier Corporation, 2004.

[11] Burghes, David N., and Alexander Graham. " Control and optimal control theories with applications." Elsevier, 2004.

[12] Doha, E. H., A. H. Bhrawy, and S. S. Ezz-Eldien. " Efficient Chebyshev spectral methods for solving multiterm fractional orders differential equations." Applied Mathematical Modelling35.12 (2011): 5662-5672.

[13] Frederico.G. S. F. and TorresD. F. M.., "Fractional optimal control in the sense of Caputo and the fractional Noether's theorem," International Mathematical Forum, vol. 3, no. 10, pp. 479-493, 2008.

[14] Frederico, Gastao SF, and Delfim FM Torres. "Noether's theorem for fractional optimal control problems." IFAC Proceedings Volumes 39.11 (2006): 79-84.

[15] Khader.M. M. " On the numerical solutions for the fractional diffusion equation." Communications in Nonlinear Science and Numerical Simulation, 16(2011):2535-2542.

[16] Meerschaert.M. M. and TadjeranC..," Finite difference approximations for two-sided space fractional partial differential equations," Appl. Numer. Math. 56 (2006), 80-90.

[17] Ozdemir, Necati, et al. "Fractional optimal control of a 2-dimensional distributed system using eigenfunctions." Nonlinear Dynamics 55.3 (2009): 251.

[18] Ozdemir.N., KaradenizD.., and I'skender.B. B., " Fractional optimal control problem of a distributed system in cylindrical coordinates," Physics Letters A, vol. 373, no. 2, pp. 221-226, 2009.

[19] Podlubny.I. "Fractional Differential Equations." Academic Press, New York, 1999.

[20] Sweilam, N. H., and M. M. Khader. " A Chebyshev pseudo-spectral method for solving fractional-order integro-differential equations." The ANZIAM Journal 51.4 (2010): 464-475.

[21] Sweilam, N. H., Al-Ajami, T. M., \& Hoppe, R. H. "Numerical solution of some types of fractional optimal control problems." The Scientific World Journal, 2013.

[22] Tricaud, Christophe, and YangQuan Chen. "Solving fractional order optimal control problems in riots 95-a generalpurpose optimal control problem solver." Proceedings of the 3rd IFAC Workshop on Fractional Differentiation and its Applications. 2008. 